\title{
Transparencia sobre los ensayos clínicos en la Región de las Américas: necesidad de coordinar las esferas regulatorias*
}

\author{
Trudo Lemmens ${ }^{1}$ y Carlos Herrera Vacaflor ${ }^{2}$
}

\begin{abstract}
Numerosas iniciativas han contribuido a aumentar la transparencia en los datos de salud de la Región de las Américas, pero se siguen necesitando nuevos esfuerzos coordinados para asegurar la fiabilidad de la investigación para la salud, según afirman Trudo Lemmens y Carlos Herrera Vacaflor
\end{abstract}

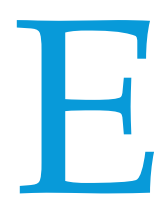
$\mathrm{n}$ su Política de investigación para la salud del 2009, la Organización Panamericana de la Salud/Organización Mundial de la Salud (OPS/OMS) reiteró el papel clave que desempeña la transparencia a la hora de asegurar que la investigación para la salud sea fiable. ${ }^{1}$ Tras varias controversias notorias, se ha promovido la transparencia especialmente en el contexto de los ensayos de productos farmacéuticos patrocinados por la industria, destinados a proporcionar datos para obtener la autorización de medicamentos por parte de las autoridades regulatorias. ${ }^{2}$ En respuesta a estas controversias, diversos interesados directos han presionado para lograr que haya un registro de los ensayos clínicos y acceso a los datos presentados a los organismos de regulación farmacéutica como herramientas clave de transparencia, con objeto de potenciar la toma de decisiones basadas en la evidencia por parte de los médicos clínicos, las autoridades regulatorias y la sociedad. ${ }^{2}$
A pesar del apoyo generalizado a la transparencia, su aplicación se enfrenta con retos importantes. La transparencia de los datos farmacéuticos se ha visto obstaculizada por la calificación de estos datos como información comercial confidencial y por las incongruencias derivadas del solapamiento de las diversas esferas regulatorias. En este artículo se abordarán en primer lugar los avances fundamentales en relación con el registro de los ensayos clínicos farmacéuticos y la difusión de los datos presentados a los organismos de regulación farmacéutica en la Región de las Américas. A continuación, los informes

\section{MENSAJES CLAVES}

- Los organismos nacionales de regulación farmacéutica involucrados en la gobernanza de la investigación relacionada con la salud deben armonizar y simplificar tanto la gobernanza del comité de ética de la investigación como los requisitos de transparencia en los diferentes sistemas regulatorios.

- Los organismos de regulación farmacéutica de los Estados Miembros de la Organización Panamericana de la Salud deben seguir el enfoque adoptado por la Agencia Europea de Medicamentos con respecto a los datos clínicos usados para tomar las decisiones de regulación farmacéutica, es decir, que estos datos no constituyen información comercial confidencial y que la carga de la prueba corresponde a las empresas farmacéuticas que son quienes deben argumentar por qué debe mantenerse la confidencialidad respecto a determinados datos.

- Los comités de ética de la investigación pueden desempeñar una función de coordinación en la promoción de las normas de transparencia en la medida en que la estructura de la gobernanza de los comités refleje su naturaleza de interés público.

\footnotetext{
* Traducción oficial al español efectuada por la Organización Panamericana de la Salud. En caso de discrepancia, prevalecerá la versión original en inglés.
}

\footnotetext{
Investigador, Facultad de Derecho, Universidad de Toronto, Canadá
} Salud Pública Dalla Lana, Universidad de Toronto, Canadá. $₫$ Trudo.Lemmens@utoronto.ca 
de tres países mostrarán la manera en que han aplicado medidas clave de transparencia y los retos que quedan por abordar. Por último, se sugerirá el papel que podría desempeñar la revisión ética de la investigación, que está presente en todas las jurisdicciones, para avanzar en la transparencia de los datos.

Los tres países elegidos, que representan a los cuatro idiomas principales en la Región de las Américas, tienen una industria farmacéutica fuerte, que se encuentra en distintas etapas de desarrollo: un mercado bien establecido (Canadá), un mercado emergente (Brasil) y un mercado emergente de segundo nivel (Argentina). La mayoría de los países latinoamericanos se encuentran en esta última categoría. Argentina y Brasil son países con un número cada vez mayor de ensayos clínicos. ${ }^{3}$ Los organismos de regulación farmacéutica de los tres países son reconocidos como autoridades regionales de referencia (recuadro 1). Dada la diversidad de la Región en cuanto al desarrollo de la industria y el examen de la regulación farmacéutica, estos estudios de casos no son exhaustivos ni plenamente representativos. Más bien, arrojan cierta luz sobre diversas iniciativas, resultados y retos existentes.

\section{AVANCES EN LATRANSPARENCIA DE LOS ENSAYOS CLÍNICOS EN LA REGIÓN DE LAS AMÉRICAS}

Durante el último decenio, la OMS, la OPS, los gobiernos nacionales, los organismos de financiamiento, las revistas científicas y algunos patrocinadores farmacéuticos de la Región de las Américas han adoptado medidas para promover la transparencia. ${ }^{2}$ El registro de ensayos clínicos, que fue la primera iniciativa que consiguió un apoyo generalizado, se exige ahora en muchos países, a pesar de que subsisten algunas brechas claras al respecto (recuadro 2). ${ }^{2}$ La OMS reconoce los registros de Brasil, Cuba y Perú como registros principales en la Región de las Américas para su Plataforma de Registros Internacionales de Ensayos Clínicos. ${ }^{5} \mathrm{Al}$ mismo tiempo, ha habido un aumento significativo del registro de ensayos en la Región. ${ }^{36}$

En lo que respecta a la difusión de los datos, ninguna autoridad nacional de regulación farmacéutica de la Región de las Américas ha seguido todavía el ejemplo de la Agencia Europea de Medicamentos, que primero facilitó el acceso a los datos en el 2010, tras lo cual en el 2014 sacó una política que partía del principio de que todos los datos de ensayos clínicos de estudios de fase 1,2 y 3 presentados en las solicitudes de autorización de medicamentos se harán públicos. ${ }^{7}$ La política del 2014 parte de la premisa de que los datos clínicos no son información comercial confidencial, y traslada la carga de la prueba a las empresas, que son quienes deben mostrar por qué es necesario mantener confidenciales ciertos componentes de esos datos. En la Región de las Américas, ni siquiera en Estados Unidos (en donde las obligaciones de revelación de los datos se establecen en la Ley de Enmiendas de la Administración de Alimentos y Medicamentos del 2007) se exige el registro de todos los ensayos clínicos, ni se publican de forma prospectiva todos los datos de los ensayos. ${ }^{8}$ Las leyes nacionales de acceso a la información de diversos países proporcionan un cierto nivel del acceso a los datos de que disponen los gobiernos, pero en esos sistemas, las autoridades de regulación farmacéutica disponen de una amplia discrecionalidad respecto a las solicitudes de acceso a los datos.

\section{BRASIL}

Desde el 2010, Brasil ha aplicado políticas de transparencia y acceso a los datos relativamente exitosas. El Registro Brasileño de Ensayos Clínicos (ReBEC) tiene como finalidad difundir la información relativa a todos los estudios en español, inglés y portugués. ${ }^{9}$ El registro en el ReBEC es obligatorio para todos los ensayos clínicos relativos a medicamentos que todavía no hayan sido autorizados oficialmente y afecta a los investigadores o participantes brasileños. La prueba del registro en la Plataforma de Registros Internacionales de Ensayos Clínicos de la OMS (ICTRP) u otro registro reconocido por el ICMJE es obligatoria para obtener la autorización de la autoridad regulatoria nacional. ${ }^{10}$ Del 2010 al 2015, se registraron en el ReBEC 3112 protocolos, ${ }^{11}$ con un incremento del registro tanto de ensayos financiados por el estado como de ensayos financiados por la industria farmacéutica. ${ }^{12}$ En los años 2016 y 2017, el número de ensayos registrados fue de 1162 y 1279, respectivamente. El ReBEC proporciona un acceso a los resúmenes de los ensayos clínicos de conformidad con el Conjunto de Datos de Registro de Ensayos Clínicos de la OMS. Los informes de los ensayos clínicos se presentan a la Agencia Nacional de Vigilancia Sanitaria de Brasil (ANVISA), pero solo puede accederse a ellos previa solicitud.

ANVISA coordina el acceso a los datos ateniéndose a lo establecido en la ley de acceso a la información de Brasil. Cualquier persona puede solicitar los datos de investigación para usarlos, reutilizarlos o redistribuirlos siempre que se cite la autoría y el origen de los datos. ANVISA ha creado un repositorio de datos de acceso libre, el Plano de Dados Abertos, que cumple las normas de calidad establecidas en la regulación actual. El acceso a los datos puede estar limitado para la protección de derechos fundamentales o del interés de la sociedad o el estado. ${ }^{13}$ Una regulación de Consejo de Salud Nacional exige que los investigadores que participan en investigaciones con financiación pública o privada publiquen sus resultados. La interrupción de la investigación y la falta de publicación deben explicarse al Comité de Ética de la Investigación (CEI) y al organismo nacional de los CEI, CONEP. ${ }^{14}$

El progreso en el fomento de la transparencia parece verse amenazado por las restricciones presupuestarias y la escasez del personal del ReBEC. ${ }^{15}$ A nivel regulatorio, la difusión de los datos puede verse más afectada aún por los acuerdos de confidencialidad de ANVISA con otros organismos de regulación.

\section{ARGENTINA}

Argentina ha elaborado políticas para la obligatoriedad del registro de los ensayos clínicos y el acceso a los datos de investigación. Sin embargo, estas iniciativas se han aplicado tímidamente, debido en parte a problemas jurisdiccionales.

Argentina aprobó el Régimen de Buena Práctica Clínica para Estudios de Farmacología Clínica (Disposición 6677/10-ANMAT), aplicable a la investigación destinada a generar datos para obtener la autorización de las autoridades regulatorias y el registro del producto. Las jurisdicciones locales también pueden establecer requisitos adicionales. Las directrices de la ANMAT mencionan la Declaración de Helsinki (versión de 1964 y versiones actuales) y el Consejo de Organizaciones Internacionales de las Ciencias Médicas (CIOMS, versión del 2002) como referencias para su aplicación, incorporando la Declaración de Helsinki y la versión del 2002 del CIOMS como parte de las buenas prácticas clínicas (BPC). Teniendo en cuenta que tanto los patrocinadores como los investigadores deben 
Recuadro 1. Organismos nacionales de regulación farmacéutica de la Región de las América

Organismos reconocidos por la OPS como referentes regionales de medicamentos y productos biológicos

- Argentina: Administración Nacional de Medicamentos, Alimentos y Tecnología Médica (ANMAT)

- Brasil: Agencia Nacional de Vigilancia Sanitaria (ANVISA)

- Canadá: Salud Canadá (HC por su sigla en inglés)

- Chile: Instituto de salud pública (ISP)

- Colombia: Instituto Nacional de Vigilancia de Medicamentos y Alimentos (INVIMA)

- Cuba: Control Estatal de Medicamentos, Equipos y Dispositivos Médicos (CEMED)

- México: Comisión Federal para Protección contra Riesgos Sanitarios de los Estados Unidos Mexicanos (COFEPRIS)

- Estados Unidos de América: Administración de Alimentos y Medicamentos (FDA por su sigla en inglés)

Organismos clasificados como evaluados y preevaluados por la OPS

- Argentina: Administración Nacional de Medicamentos, Alimentos y Tecnología Médica (ANMAT)

- Bahamas: Organismo Nacional de Medicamentos de las Bahamas (BNDA por su sigla en inglés)

- Barbados: Servicio de Medicamentos de Barbados (BDS por su sigla en inglés)

- Bolivia: Unidad de Medicamentos y Tecnologías Sanitarias (UNIMED)

- Brasil: Agencia Nacional de Vigilancia Sanitaria, Ministerio de Salud (ANVISA)

- Canadá: Salud Canadá (HC por su sigla en inglés)

- Chile: Instituto de Salud Pública (ISP)

- Costa Rica: Consejo Nacional de Investigación en Salud (CONIS), CEI de universidades, Caja Costarricense de Seguro Social (CCSS)

- Cuba: Control Estatal de Medicamentos, Equipos y Dispositivos Médicos (CEMED)

- República Dominicana: Dirección General de Medicamentos, Alimentos y Productos Sanitarios (DIGEMAPS)

- Ecuador: Instituto Nacional de Higiene y Medicina Tropical, Ministerio de Salud

- El Salvador: Dirección Nacional de Medicamentos

- Guatemala: Departamento de Regulación y Control de Productos Farmacéuticos y Afines (MEDICAMENTOS)

- Guyana: Departamento de Alimentos y Medicamentos (FDD por su sigla en inglés)

- Haití: Dirección de Farmacia, Medicamentos y Medicina Tradicional (DPMMT por su sigla en francés)

- Honduras: Secretaría de Salud, Dirección General de Regulación Sanitaria

- Jamaica: División de Normas y Reglamentación (DSR por su sigla en inglés)

- México: Comisión Federal para Protección contra Riesgos Sanitarios de los Estados Unidos Mexicanos (COFEPRIS)

- Panamá: Dirección Nacional de Farmacia y Drogas

- Paraguay: Dirección Nacional de Vigilancia Sanitaria

- Perú: Dirección General de Medicamentos, Insumos y Drogas (DIGEMID)

- Suriname: Autoridad Nacional de Regulación

- Trinidad y Tabago: Organismo Nacional de Regulación Farmacéutica

- Estados Unidos de América: Administración de Alimentos y Medicamentos (FDA por su sigla en inglés)

- Venezuela: Instituto Nacional de Higiene Rafael Rangel (IHRR)

Nota: Todos los organismos mencionados actúan como parte de los ministerios de salud nacionales. En el Caribe de habla inglesa, el Organismo de Salud Pública del Caribe (CARPHA) de la OPS se encarga de establecer normas uniformes. También está la Red Panamericana para la Armonización de la Reglamentación Farmacéutica de la OPS (Red PARF).

respetar estas directrices internacionales, la referencia a diferentes directrices y diferentes versiones en regímenes de gobernanza solapados -no exclusivos de Argentina- puede plantear problemas de compatibilidad y uniformidad. Las obligaciones éticas actuales, más detalladas, respecto a la transparencia de la investigación relacionada con la salud establecidas en la versión del 2016 del CIOMS no forman parte todavía de las BPC de Argentina.

Argentina creó su Registro Nacional de Investigaciones en Salud (RENIS) para aumentar el registro de los ensayos clínicos y otras investigaciones para la salud. ${ }^{16}$ La investigación para la salud financiada por el Ministerio de Salud o realizada bajo las normas de la Administración Nacional de Medicamentos, Alimentos y Tecnología Médica (ANMAT) debe registrarse para ser autorizada. La aplicación de requisitos de registro de otros ensayos clínicos llevados a cabo en las provincias depende de las autoridades sanitarias locales. El RENIS también contiene información sobre los comités de ética de la investigación, los patrocinadores, los investigadores y las organizaciones de investigación bajo contrato. En los años 2016 y 2017, el RENIS registró 145 y 180 proyectos de investigación,, ${ }^{17}$ respectivamente, mientras que se ingresaron $191 \mathrm{y}$ 125 ensayos clínicos en la base de datos de la ANMAT para estudios de farmacología clínica ${ }^{18}$ en esos mismos períodos. Las directrices de la ANMAT contienen normas respecto a la confidencialidad de los pacientes y la presentación de los datos (como informes de ensayos clínicos) a la ANMAT, pero no al público.

Hay diversas leyes que afectan al acceso a los datos de investigación. Las leyes que rigen el acceso a la información exigen que el gobierno, el estado y los organismos descentralizados proporcionen acceso a los datos que estén bajo su control. ${ }^{19}$ Una Ley de Protección de los Datos Personales o hábeas data reglamenta también el acceso a los datos. ${ }^{20}$ Hay obligaciones de privacidad adicionales en 
lo que respecta a los datos personales. Sin embargo, si los datos no son identificables, no puede negarse el acceso a ellos si dicho acceso tiene fines científicos o es en interés público. Los datos inidentificables también pueden divulgarse y transferirse internacionalmente sin necesidad de un consentimiento para ello. ${ }^{21}$ La ley considera los datos de seguridad y eficacia presentados a la ANMAT como secretos comerciales o datos comerciales. ${ }^{22}$ Esto puede crear dificultades para aplicar la divulgación de los datos a los investigadores independientes y al público.

Argentina es uno de los países con una tasa de registro de ensayos clínicos más alta por población, ${ }^{3}$ lo cual pone de manifiesto el gran valor del esfuerzo realizado con el RENIS. Aunque el registro muestre los datos de resumen de los ensayos clínicos, el RENIS no cumple la norma del conjunto de datos de la OMS, ni proporciona acceso a los datos en los

Recuadro 2. Situación de los Estados Miembros de la OPS

Estados con requisitos de registro formales y obligatorios de los ensayos clínicos*

- Brasil

- Colombia

- El Salvador

- Estados Unidos

- Guatemala

- Panamá

- Perú

- Uruguay

Estados con registros nacionales $†$

- Argentina

- Brasil

- Cuba

- Estados Unidos

- México

- Perú

* Compilación basada en una encuesta de la OMS sobre los perfiles farmacéuticos nacionales en la Región de las Américas, con comprobación con la información presentada por los países respecto a las políticas actuales en las páginas web de los organismos de reglamentación nacionales. ${ }^{4}$ Incluye tan solo los países en los que es obligatorio el registro en su estructura de regulación farmacéutica. Algunos países (como Canadá) tienen requisitos de registro parciales mediante directrices del organismo de financiamiento que forman parte también de las buenas prácticas clínicas.

† Los registros de Argentina, Estados Unidos y México no son reconocidos como registros principales de la OMS. ${ }^{3}$ idiomas de la OPS (francés, inglés y portugués) aparte del español. ${ }^{23}$ Esto puede impedir la visibilidad de la investigación local para atraer la realización de ensayos internacionales. ${ }^{9}$ Además, el acceso a los datos de investigación está regulado en múltiples marcos jurídicos distintos. Esto crea confusión con respecto al derecho de los investigadores y de las organizaciones de la sociedad civil de acceder a los datos.

\section{CANADÁ}

Canadá no tiene un registro integral propio de los ensayos clínicos. La base de datos de ensayos clínicos del Ministerio de Salud de Canadá [Health Canada] suministra información sobre los ensayos clínicos, pero tiene como principal objetivo estimular el reclutamiento en las investigaciones. ${ }^{24} \mathrm{El}$ registro de ensayos clínicos y la presentación pública de los resultados son obligatorios en los centros con financiamiento federal por las normas de ética de investigación de los organismos de financiamiento federales, la declaración de políticas de los tres consejos sobre ética de la investigación con seres humanos (conocida como TCPS2 por su sigla en inglés $)^{25}$ y los requisitos del organismo de financiamiento específico. ${ }^{26}$ Sin embargo, la Dirección General de Productos de Salud y Alimentos del Ministerio de Salud de Canadá, que es el organismo federal de regulación farmacéutica, no exige de manera explícita el registro de los ensayos clínicos de medicamentos y productos sanitarios. Las directrices de buenas prácticas clínicas del Ministerio de Salud de Canadá ${ }^{27}$ hacen referencia a los principios de la Declaración de Helsinki como parte de los antecedentes de las buenas prácticas clínicas y el propio CEI del Ministerio sigue lo establecido en la TCPS2. Tanto la Declaración de Helsinki como la TCPS2 exigen el registro de los ensayos clínicos y la presentación de los resultados. Estas referencias indirectas no establecen una exigencia legal de su cumplimiento, aunque tal vez podrían interpretarse como parte integrante de las buenas prácticas clínicas. Los informes oficiales han subrayado durante mucho tiempo la necesidad de mejorar la transparencia en el registro regulatorio de los ensayos clínicos. ${ }^{28}$ Los ensayos tienden a registrarse en el registro de la FDA de Estados Unidos, y la mayor parte de las revistas médicas de Canadá, si no todas, exigen también un registro previo.
La legislación sobre el acceso a la información puede usarse para solicitar el acceso a los datos, pero el Ministerio de Salud de Canadá ha insistido en el pasado en que los solicitantes muestren de qué modo su necesidad de información es superior al posible daño comercial causado a la empresa que presenta los datos, lo cual dificulta la transparencia de los datos. Una enmienda del 2014 a la ley de alimentos y medicamentos establece una base legal para la revelación de los datos de los ensayos clínicos sin el consentimiento del patrocinador una vez que un medicamento ha sido autorizado, y podría usarse para otras regulaciones adicionales acerca de registro de los ensayos. ${ }^{29}$ No obstante, la ley hace referencia a los datos de los ensayos clínicos como información comercial confidencial, lo cual socava aparentemente el concepto de los datos como un bien público. Actualmente, el Ministerio de Salud de Canadá exige también la firma de un acuerdo de confidencialidad antes de proporcionar acceso a los datos, lo cual retrasa este acceso y la posterior divulgación de los datos. ${ }^{30}$ Sin embargo, una propuesta preliminar de regulación ${ }^{31}$ indica un posible cambio en el enfoque regulatorio acerca de la transparencia respecto a los datos. Aparentemente inspirada en el enfoque de la Agencia Europea de Medicamentos, la propuesta preliminar de regulación sostiene que los resúmenes clínicos, informes y datos de apoyo de los ensayos clínicos presentados en el proceso de evaluación de medicamentos no se considerarán información comercial confidencial después de que haya habido una decisión final del organismo de regulación farmacéutica. Esto trasladaría la carga de la prueba a los patrocinadores farmacéuticos que serían quienes tendrían que mostrar por qué la autoridad farmacéutica debe mantener la confidencialidad respecto a datos concretos. Esta regulación no se manifiesta respecto a la exigencia de que los investigadores firmen un acuerdo de confidencialidad, una práctica que puede continuar entorpeciendo el acceso a los datos y su divulgación. La propuesta de regulación no aborda tampoco la cuestión del registro de los ensayos, que requeriría otra regulación nueva aparte.

\section{RETOS PARA LA APLICACIÓN Y ESTRATEGIAS REGIONALES}

Un reto fundamental para una estrategia de transparencia de datos coherente, 
en particular en lo que respecta al acceso a los datos de los ensayos clínicos de productos farmacéuticos, es el hecho de que haya sistemas regulatorios distintos, aunque solapados, que determinan si se pueden divulgar los datos y en qué medida es posible hacerlo. La mayoría de los países tienen directrices específicas respecto a la ética en la investigación con financiamiento público. Inspirándose en las directrices internacionales, como la Declaración de Helsinki ${ }^{32}$ a la que se hace referencia a menudo y el Consejo de Organizaciones Internacionales de las Ciencias Médicas, ${ }^{33}$ es cada vez más frecuente que se incluya un mayor grado de obligaciones de transparencia como las de registro y presentación de un resumen de los resultados.

En países como Canadá, los ensayos clínicos farmacéuticos con financiamiento comercial que se realizan en instituciones con financiamiento federal deben cumplir estas directrices. Las regulaciones relativas a alimentos y medicamentos, incluidos los requerimientos de buenas prácticas clínicas, hacen referencia a menudo a las directrices de ética internacionales. ${ }^{32}$ No obstante, los patrocinadores de la industria farmacéutica insisten en que los datos de los ensayos clínicos constituyen información comercial confidencial, algo que los sistemas regulatorios a menudo aceptan. Esto crea impedimentos para la difusión de los datos, si bien el acceso a los sistemas de información permite a menudo a los investigadores solicitar el acceso a los datos de regulación farmacéutica tras la autorización del producto. No obstante, en estos casos, los organismos de regulación farmacéutica tienden a ejercer cierta discrecionalidad $\mathrm{y}$, a menudo, se ven presionados por la insistencia de la industria respecto a la confidencialidad de los datos.

Los CEI podrían desempeñar un papel importante al insistir en el acceso a los datos como un requisito ético fundamental y conseguir que se agreguen compromisos con respecto a la transparencia en los formularios de consentimiento de los participantes en la investigación. Garantizar el consentimiento individual para la divulgación de los datos puede ayudar también a enfrentar las posibles inquietudes acerca de la privacidad de la información de salud personal que se encuentra en los datos de los ensayos clínicos. Está ampliamente reconocido, incluso en las normas de buenas prácticas clínicas internacionales, que los CEI tienen obligaciones clave en cuanto a la protección de los derechos, la seguridad y el bienestar de los participantes en la investigación. Si, como reconocen las directrices de ética nacionales e internacionales, la transparencia es un componente fundamental de la investigación ética, los CEI deberían requerir que los investigadores y los patrocinadores acepten compromisos específicos de transparencia como condición para obtener la autorización en cuanto a la ética. Además, teniendo en cuenta que cada vez se reconoce más el papel de los CEI después de la autorización de los medicamentos, cabe argumentar que los CEI deberían verificar de forma activa y, en la mayor medida posible, hacer cumplir las normas de transparencia. ${ }^{34}$

Reconocemos las dificultades existentes para lograrlo. Incluso en Europa, donde la transparencia enfrenta menos obstáculos regulatorios, los CEI carecen de procedimientos adecuados para verificar la publicación de los resultados o reducir al mínimo el uso de una presentación selectiva de la información. ${ }^{35}$ En la Región de las Américas, las diferencias existentes en las normas principales sobre transparencia en la investigación entre los distintos países se reflejan también en diferencias en los sistemas de gobernanza de los CEI. Algunos países disponen de una estructura administrativa coherente y centralizada con normas uniformes para los CEI, que pueden facilitar el cumplimiento de las normas de transparencia. Por ejemplo, en Brasil, todos los CEI de centros de investigación son responsables de examinar los ensayos llevados a cabo en su centro. Sin embargo, el CONEP examina las decisiones de los CEI y puede solicitar cambios. También autoriza, registra y supervisa la actividad de los CEI de centros de investigación. Aunque la estructura de Brasil establece una rendición de cuentas pública y un examen coherente de los CEI, el CONEP continúa estando insuficientemente financiado. ${ }^{9}$

Los CEI para los ensayos promovidos por la industria en otras jurisdicciones siguen una estructura más orientada al mercado. En países como Canadá (en algunas de las principales provincias), Estados Unidos y Argentina (excepto en la provincia y la ciudad de Buenos Aires), ${ }^{36}$ la mayor parte de los ensayos promovidos por la industria son examinados por CEI comerciales. Estos CEI tienen una relación directa de cliente-proveedor con los patrocinadores de la industria y compiten por el negocio que la industria genera. ${ }^{37}$ Son interesados directos en el sector de producción de conocimiento que prestan apoyo a las industrias farmacéuticas y de productos sanitarios, y actúan bajo las mismas normas del mercado comercial (incluidas las formas de confidencialidad comercial). ${ }^{38}$ La gobernanza de los CEI de orientación comercial para la investigación promovida por la industria parece poco apropiada para exigir el cumplimiento y fomentar el registro de los ensayos y la divulgación de los datos. Las cuestiones jurisdiccionales pueden crear obstáculos adicionales para los intentos de promover una estructura de los CEI con responsabilidad pública. Esto es lo que sucede, por ejemplo, en Argentina y Canadá. En cada provincia argentina, los CEI de centros de investigación y comerciales son coordinados por CEI centrales. La resolución 1002/2016 creó el Comité Nacional Asesor de Ética en Investigación, que colabora con los CEI provinciales y promueve la coordinación. Su mandato incluye el registro de los CEI que actúan en centros nacionales o en organismos descentralizados del Ministerio de Salud; sin embargo, no abarca los CEI privados. En un reciente estudio de caso de Argentina sobre los ensayos promovidos por la industria, se ha documentado que el gobierno y un CEI comercial no fueron capaces de exigir el cumplimiento de normas de transparencia básicas. ${ }^{39}$ En Canadá tampoco hay regulación federal ni supervisión federal de los CEI. ${ }^{40}$ Las provincias más pobladas, que son las que tienen un mayor grado de actividad de ensayos clínicos farmacéuticos, es decir, Ontario y Quebec, se basan en gran parte en CEI comerciales privados para el examen de los ensayos clínicos farmacéuticos. Tan solo una provincia, Terranova y Labrador, ha aprobado legislación que encarga explícitamente a una autoridad central de ética de investigación para la salud la organización del examen de toda la investigación realizada en la provincia por parte de un CEI central. ${ }^{41}$

\section{CONCLUSIONES}

Los países de la Región de las Américas han adoptado medidas importantes para aplicar normas de transparencia. Algunos de ellos han realizado avances importantes en la aplicación del registro 
obligatorio de los ensayos clínicos y de un cierto grado de transparencia. No obstante, algunos países líderes continúan sin exigir la inclusión en un registro de acceso público. Aunque se han emprendido iniciativas de divulgación de los datos en varios países, la transparencia sobre los datos de ensayos clínicos farmacéuticos se ha visto obstaculizada por su consideración como información comercial confidencial. No obstante, la mayor parte de jurisdicciones reconocen el fundamento ético sustancial que tiene la transparencia en cuanto a los datos, a menudo haciendo referencia explícita a las directrices internacionales de ética de la investigación. Esto se refleja también en los enfoques de derechos humanos y del derecho público a la divulgación de los datos. ${ }^{38}$ Si los países de la Región quisieran simplificar los diferentes ámbitos regulatorios solapados que rigen el uso de los datos de los ensayos clínicos y seguir el camino emprendido por la Agencia Europea de Medicamentos de establecer la presunción de que los datos deben ser de acceso público, tendrían que tomar medidas claras destinadas a la aplicación de una transparencia integral respecto a los datos.

Para aplicar una transparencia coherente respecto a los datos de salud en la Región de las Américas, los países tienen que hacer malabarismos con las diferentes regulaciones, directrices y herramientas de gobernanza aplicables. Esto significa que diferentes organismos regulatorios y organismos de financiamiento coordinan los regulaciones con respecto a la transparencia aplicables en cada nivel nacional. Se necesita un mayor desarrollo de los sistemas de CEI con responsabilización pública, que rindan cuentas directamente ante el estado, como parte de las medidas destinadas a promover una transparencia significativa.

Agradecimientos. A Luis Salicrup y Luis Gabriel Cuervo, editores de The BMJ, y a los revisores sus comentarios sobre el artículo, y a Suzanne Stephens la corrección del texto. Agradecemos el apoyo económico del Programa de Contribuciones de la Oficina del Comisionado de Privacidad de Canadá, para un proyecto sobre el Acceso a Datos de Ensayos Clínicos y la Privacidad.

Contribución de los autores. Ambos autores declaran que han realizado contribuciones sustanciales a la concepción y el diseño del trabajo; a la adquisición, el análisis o la interpretación de los datos para este trabajo; y en la redacción del artículo y su revisión crítica. Los dos han aprobado la versión final y están de acuerdo con ser considerados responsables por su contenido y por garantizar que cualquier pregunta relacionada con la precisión o la integridad de alguna parte de este trabajo sea investigada y resuelta adecuadamente

Conflicto de intereses. Hemos leído y comprendido la política de The BMJ sobre el conflicto de intereses y no tenemos intereses pertinentes que declarar.

Procedencia del artículo y revisión por pares. Comisionado; revisión por pares externa.

Declaración. Las opiniones expresadas en este manuscrito son responsabilidad del autor y no reflejan necesariamente los criterios ni la política de la RPSP / PAJPH y/o de la OPS/OMS.

Serie Fortalecimiento de la investigación para la salud en las Américas. Este artículo es parte de una serie propuesta por la Organización Panamericana de la Salud (OPS) y comisionada por The BMJ, que tuvo a cargo la revisión por pares y la edición, y tomó la decisión sobre la publicación de los artículos sin participación de la OPS. Los gastos de publicación en acceso abierto de la serie original en inglés fueron financiados por la OPS. Acceso a la serie original en inglés en www.bmj.com/health-research-americas; acceso a la serie en español y portugués en https://www.paho.org/journal/es /numeros-especiales / fortalecimiento -investigacion-para-salud-americas.

\section{REFERENCIAS}

1. Organización Panamericana de la Salud/ Organización Mundial de la Salud. Política de Investigación para la Salud. Documento CD49/10, 49. ${ }^{\circ}$ Consejo Directivo, $61 .{ }^{a}$ sesión del Comité Regional de la OMS para las Américas. 2009. http://www1.paho.org /hq/dmdocuments/2009/CD49-10-s.pdf.

2. Krleža-Jeriç K, Lemmens $T$, Reveiz L, Cuervo LG, Bero LA. Prospective registration and results disclosure of clinical trials in the Americas: a roadmap toward transparency. Rev Panam Salud Publica. 2011;30:87-96.

3. Rodríguez-Feria P, Cuervo LG. Progress in trial registration in Latin America and the Caribbean, 2007-2013. Rev Panam Salud Publica. 2017;41:e31.

4. Organización Mundial de la Salud. Pharmaceutical sector country profiles data and reports. Región de las Américas. OPS, 2010. www.who.int/medicines/areas/co ordination/coordination_assessment/en /index1.html.

5. Registro Peruano de Ensayos Clínicos. El REPEC ahora es un registro primario de la OMS. 21 Febrero del 2017. www .ensayosclinicos-repec.ins.gob.pe/86-slide show / 270-el-repec-ahora-es-un-registro -primariode-la-oms.

6. White L, Ortiz Z, Cuervo LG, Reveiz L. Clinical trial regulation in Argentina: overview and analysis of regulatory framework, use of existing tools, and researchers' perspectives to identify potential barriers. Rev Panam Salud Publica. 2011;30:445-52.

7. Agencia Europea de Medicamentos. External guidance on the implementation of the European Medicines Agency Policy on publication of clinical data for medicinal products for human use. 12 de abril del 2017. www.ema.europa.eu/ema/index .jsp?curl=pages/regulation/general/gene ral_content_001799.jsp\&mid=WC0b01ac 0580b2f6ba.

8. Administración de Alimentos y Medicamentos de Estados Unidos. FDA 801 requirements. 2017. https://clinicaltrials .gov/ct $2 /$ manage-recs/fdaaa.

9. Freitas CBD, Hossne WS, Dutra S. Progress and challenges of clinical research with new medications in Brazil. En: Homedes $\mathrm{N}$, Ugalde A, eds. Clinical trials in Latin
America: where ethics and business clash Springer. 2014:151-71

10. Agencia Nacional de Vigilancia Sanitaria de Brasil. Perguntas e responstas RDC 9/2015: resolução RDC No 9 art.2-3 (2015). 2015. http://portal.anvisa.gov.br /documents/33836/2492465/Perguntas+e + respostas+sobre+a+RDC+09+de+2015 /b14fa962-c1e9-41f5-9524-a290c5b4e98e.

11. Organización Panamericana de la Salud/ Organización Mundial de la Salud. Relatório de gestão dos termos de cooperação 2015 [Cooperation Terms Management Report 2015]. 2015. www.paho.org/bra /images/relatorio_gestao_2_sem_2015_o16 .pdf?ua $=1$.

12. Silva LR, Laguardia J, Bastos Alves MRA, et al. ReBEC em números: reflexos da política mandatória em pesquisa clínica na trajetória do Registro Brasileiro de Ensaios Clinicos. Cadernos BAD. 2014;2 juldez:107-14. https://www.bad.pt/public acoes/index.php/cadernos/article/view $/ 1187$.

13. Agencia Nacional de Vigilancia Sanitaria de Brasil. Sobre a Lei de Acesso a 
Informação. 2011. http:/ / portal.anvisa.gov br/sobre-a-lei-de-acesso-a-informcao.

14. Ferreira da Silva C, Ventura M, Castro C. Bioethical perspective of justice in clinical trials. Rev Bioet 2016;24:292-303.

15. Freitas CG, Pesavento TFC, Pedrosa MR, et al. Practical and Conceptual issues of Clinical Trial Registration for Brazilian Researchers. Sao Paulo Med J. 2016; 134:28-33.

16. Argentina. Resolución 1480/2011. Apruébase la guía para investigaciones con seres humanos. Bs.As., 13 de septiembre del 2011. http://servicios.infoleg.gob.ar/infoleg Internet/anexos/185000-189999/187206 /norma.htm

17. Argentina, Ministerio de Salud. ReNIS: Registro Nacional de Investigaciones en Salud. www.argentina.gob.ar/salud/regis troinvestigaciones.

18. Estudios Clinicos. www.anmat.gov.ar /aplicaciones_net/applications/consultas /ensayos_clinicos/Consulta_EC.asp\#.

19. Ministerio de Justicia y de Derechos Humanos de Argentina. InfoLEG: Información Legislativa. Ley 27275: Derecho de Acceso a la Información Pública, 29 Septiembre 2016. http:/ / servicios.infoleg.gob.ar/infoleg Internet/anexos/265000-269999/265949 /norma.htm.

20. Argentina. Ministerio de Justicia y de Derechos Humanos de Argentina. InfoLEG: Información Legislativa. Ley 25.326: Protección de Datos Personales. 30 Oct 2000. Buenos Aires: InfoLEG; 2016. http:// servicios.infoleg.gob.ar/infolegInternet /anexos / 60000-64999/64790/texact.htm

21. Outomuro D, Mirabile LM. Confidencialidad y Privacidad en la medicina y en la investigación científica: desde la bioética a la ley. Rev Bioet. 2015;23:238-43. www .scielo.br/pdf/bioet/v23n2/1983-8034 -bioet-23-2-0238.pdf doi:10.1590/1983 $-80422015232062$.

22. Ministerio de Justicia y de Derechos Humanos de Argentina. InfoLEG: Información Legislativa. Ley de confidencialidad sobre información y productos que estén legítimamente bajo control de una persona y se divulgue indebidamente de manera contraria a los usos comerciales honestos: Ley 24-766, 20 dic 1996. Buenos Aires: InfoLEG. 1996. http://servicios.infoleg.gob.ar/info legInternet/anexos/40000-44999/41094 /norma.htm

23. Organización Panamericana de la Salud/ Organización Mundial de la Salud. Advisory committee on health research: a review of its contributions to health and research for health in the Americas 2009-2015. 2016. www.paho.org/hq/index.php?option=com _docman\&task=doc_download\&gid=36641 \&Itemid $=270$.

24. Lemmens T, Gibson S. Decreasing the data deficit: improving post-market surveillance in pharmaceutical regulation. McGill Law J 2014;59:943-88 doi:10.7202/ 1026134ar.

25. Canadian Institutes of Health Research, Natural Sciences and Engineering Research Council of Canada, and Social Sciences and Humanities Research Council of Canada. Tri-council policy statement: ethical conduct for research involving humans. 9 de diciembre del 2014. www.pre.ethics.gc.ca /pdf/eng/tcps2-2014/TCPS_2_FINAL_ Web.pdf.

26. Canadian Institutes of Health Research. Section 2: grants and awards management-CIHR's grants and awards guide. 2013. www.cihr-irsc.gc.ca/e/22631.html\#2 -A20.

27. Health Canada. Notice: ICH guideline E6: good clinical practice: consolidated guideline. 2004. www.hc-sc.gc.ca/dhp-mps /alt_formats/hpfb-dgpsa/pdf/prod pharma/e6-eng.pdf.

28. Standing Senate Committee on Social Affairs, Science, and Technology. Canada's clinical trial infrastructure: a prescription for improved access to new medicines. 2012. www.aihealthsolutions.ca/media/Senate -report.pdf.

29. Herder M. The opacity of Bill C-17's transparency amendments. Impact Ethics Blog 23 de junio del 2014. https://impactethics .ca/2014/06/23/the-opacity-of-bill-c-17s -transparency-amendments/.

30. Herder $\mathrm{M}$, Lemmens $\mathrm{T}$, Lexchin $\mathrm{J}$, et al. Pharmaceutical transparency in Canada: tired of talk. BMJ Blog 6 de junio del 2016. http:/ /blogs.bmj.com/bmj/2016/06/06 /pharmaceutical-transparency-in-canada -tired-of-talk.

31. Gobierno de Canadá, Departamento de Salud. Regulations amending the Food and Drug Regulations (public release of clinical information). 9 de diciembre del 2017. http://gazette.gc.ca/rp-pr/p1/2017 /2017-12-09/html/reg3-eng.html.

32. Asociación Médica Mundial. Declaración de Helsinki de la AMM - principios éticos para las investigaciones médicas en seres humanos. 2013. https://www .wma.net/es/policies-post/declaracion -de-helsinki-de-la-amm-principios-eticos -para-las-investigaciones-medicas-en-seres -humanos/.
33. Consejo de Organizaciones Internacionales de las Ciencias Médicas. Pautas éticas internacionales para la investigación relacionada con la salud con seres humanos. 2016. https://cioms.ch/wp-content/ uploads / 2017/12/CIOMS-EthicalGuideline_SP_INTERIOR-FINAL.pdf

34. Kolstoe SE, Shanahan DR, Wisely J. Should research ethics committees police reporting bias? BMJ. 2017;356:j1501. PubMed doi:10.1136/bmj.j1501

35. Strech D, Littmann J. The contribution and attitudes of research ethics committees to complete registration and non-selective reporting of clinical trials: a European survey. Res Ethics Rev. 2016;12:123-36 doi:10 $.1177 / 1747016115626497$

36. Sabio MF, Bortz JE. Estructura y funcionamiento de los comités de ética en investigación de la Ciudad Autónoma de Buenos Aires y el Gran Buenos Aires. Salud Colect. 2015;11:247-60. http:/ /www.scielosp .org/pdf/scol/v11n2/v11n2a08.pdf. doi:10 $.18294 / \mathrm{sc} .2015 .687$

37. Lemmens T, Freedman B. Ethics review for sale? Conflict of interest and commercial research review boards. Milbank Q. 2000;78:547-84, iii-iv. doi:10.1111/1468 $-0009.00185$

38. Lemmens T. Pharmaceutical knowledge governance: a human rights perspective. J Law Med Ethics. 2013;41:163-84. doi:10 $.1111 /$ jlme. 12012

39. Homedes N, Ugalde A. The evaluation of complex clinical trial protocols: resources available to research ethics committees and the use of clinical trial registries-a case study. J Med Ethics. 2015;41:464-9. doi:10 .1136/medethics-2013-101381

40. Lemmens T. Federal regulation of REB review of clinical trials: a modest but easy step towards an accountable REB review structure in Canada. Health Law Rev. 2005;13:39-50.

41. Newfoundland and Labrador: health research ethics authority act. http:// assembly.nl.ca/Legislation/sr / statutes /h01-2.htm

Forma de citar (artículo original): BMJ 2018;362:k2493 doi: http://dx.doi.org/10.1136/bmj.k2493

Manuscrito original en inglés publicado el 16 de julio de 2018. 